\title{
High attrition before and after ART initiation among youth (15-24 years of age) enrolled in HIV care
}

\section{Matthew R. Lamb ${ }^{\mathrm{a}, \mathrm{b}}$, Ruby Fayorsey ${ }^{\mathrm{a}}$, Harriet Nuwagaba-Biribonwoha ${ }^{\mathrm{a}}$, Violante Viola ${ }^{\mathrm{a}}$, Vincent Mutabazi $^{\mathrm{c}}$, Teresa Alwar ${ }^{\mathrm{a}}$, Caterina Casalini ${ }^{\mathrm{a}}$, and Batya Elul ${ }^{\mathrm{a}, \mathrm{b}}$}

aCAP-Columbia University, New York, USA bepartment of Epidemiology, Mailman School of Public Health, Columbia University, New York, New York, USA 'Rwanda Biomedical Center, Kigali, Rwanda

\section{Abstract}

Objectives-To compare pre and post-ART attrition between youth (15-24 years) and other patients in HIV care, and to investigate factors associated with attrition among youth.

Design-Cohort study utilizing routinely collected patient-level data from $160 \mathrm{HIV}$ clinics in Kenya, Mozambique, Tanzania, and Rwanda.

Methods-Patients at least 10 years of age enrolling in HIV care between 01/05 and 09/10 were included. Attrition (loss to follow-up or death 1 year after enrollment or ART initiation) was compared between youth and other patients using multivariate competing risk (pre-ART) and traditional (post-ART) Cox proportional hazards methods accounting for within-clinic correlation. Among youth, patient-level and clinic-level factors associated with attrition were similarly assessed.

Results-A total of 312335 patients at least 10 years of age enrolled in HIV care; 147 936(47\%) initiated ART, 17\% enrolling in care and 10\% initiating ART were youth. Attrition before and after ART initiation was substantially higher among youth compared with other age groups. Among youth, nonpregnant women experienced lower pre-ART attrition than men [sub-division hazard ratio $=0.90,95 \%$ confidence interval $(\mathrm{CI}): 0.86-0.94$ ], while both pregnant [adjusted hazard ratio $(\mathrm{AHR})=0.85,95 \% \mathrm{CI}: 0.74-0.97$ ] and nonpregnant $(\mathrm{AHR}=0.79,95 \% \mathrm{CI}: 0.73-0.86)$ female youth experienced lower post-ART attrition than men. Youth attending clinics providing sexual and reproductive health services including condoms (AHR $=0.47,95 \% \mathrm{CI}: 0.32-0.70$ ) and clinics offering adolescent support groups (AHR $=0.73,95 \%$ CI: $0.52-1.0$ ) experienced significantly lower attrition after ART initiation.

Correspondence to Matthew Lamb, ICAP-Columbia University, 60 Haven, Room B-112 New York, NY 10032, USA. Tel: +1 212 304 7135; mrl2013@columbia.edu.

Conflicts of interest: The authors have no conflicts of interest to declare.

Preliminary analysis of data included in this article was presented at the 19th Conference on Retroviruses and Opportunistic Infections. Factors associated with high loss to follow-up among 15- to 24-year-olds enrolled in HIV care: sub-Saharan Africa (Paper \#1149). Seattle, Washington. March 5-8, 2012. 
Conclusion-Youth experienced substantially higher attrition before and after ART initiation compared with younger adolescents and older adults. Adolescent-friendly services were associated with reduced attrition among youth, particularly after ART initiation.

\section{Keywords}

adolescents; AIDS; attrition; cohort study; epidemiology; HIV; HIV/AIDS; loss to follow-up; PEPFAR; retention; sub-Saharan Africa; young adults; youth

\section{Introduction}

Currently, an estimated four million HIV-infected youth aged 15-24 years reside in subSaharan Africa [1], and account for $20 \%$ of all people living with HIV/AIDS in the region [2]. With approximately $40 \%$ of all new HIV infections occurring among youth [3] and perinatally infected children on antiretroviral therapy (ART) aging into this group [4], this population is expected to grow. As young people are critical to the economic future of subSaharan Africa, optimizing outcomes across the HIV care cascade among HIV-infected youth is a priority [5].

The transition from childhood through adolescence to adulthood often involves rapid, sometimes destabilizing, change that may compromise these outcomes. Youth may leave parental supervision to form their own families, and/or migrate for work or education. They may make healthcare decisions for themselves for the first time, often without sufficient knowledge of the importance of sustained engagement with healthcare systems. The effect of these and the other psychosocial, cognitive and behavioral changes that characterize this period is reflected in the emerging literature on HIV-related outcomes among young people. Several studies in sub-Saharan Africa have shown that when compared with adults 25 years and older, youth are substantially less likely to know their HIV status [6-9], and youth in sub-Saharan Africa have been found to lack basic knowledge about HIV/AIDS transmission and prevention $[1,10]$. Published data on treatment outcomes among adolescents and youth on ART also show worse adherence, retention and survival in these groups compared with older adults [11-17].

Very little information is available, however, on outcomes among youth enrolled in HIV care who are not yet eligible for ART. Data are also lacking on the role services targeting young people play in improving outcomes among youth in HIV care and on ART. We used routinely collected patient-level and clinic-level data from a large, multicountry service delivery program in sub-Saharan Africa to compare attrition before and after ART initiation between youth and younger adolescents and older adults, and investigated factors associated with attrition among youth.

\section{Methods}

\section{Study population}

The study population included patients aged at least 10 years who enrolled in HIV care between January 1, 2005 and September 30, 2010 at 160 HIV care and treatment clinics in four sub-Saharan African countries: Kenya (41 clinics), Mozambique (31), Rwanda (41), 
and Tanzania (47). Patients were followed up until September 30, 2011. 'Enrolling into HIV care' is defined as the date in which a patient, after testing HIV-positive, attends an HIV care clinic for a visit and that date is recorded on the enrollment form. Information is not available on the proportion of patients testing HIV-positive at a given clinic who subsequently enroll into HIV care. All clinics were receiving technical and financial support from ICAP at Columbia University through funding from PEPFAR. Provision of services at each clinic was governed by national guidelines.

\section{Data sources}

Patient-level data-Patient information routinely collected during each clinic visit (described in Table 1) was documented by clinicians on national patient forms. Information from these forms was regularly entered into on-site electronic databases by data clerks. Information is collected at enrollment and subsequent follow-up visits, with visit frequency depending on patient immunologic status and whether they have initiated ART. Data quality assessments were done at least annually at each clinic. Each quarter, these data were deidentified at the site-level, encrypted and transferred to the ICAP-Columbia University office in-country, and imported into a common-format database maintained in ICAPColumbia University's New York office. Use of these de-identified patient-level data for research purposes has been approved by the Columbia University Medical Center IRB and ethical review boards in each of the four countries.

Clinic-level data-Data on clinic characteristics were derived cross-sectionally from structured assessments completed by ICAP staff in consultation with clinical employees in August 2011 and applied retrospectively. These assessments captured information regarding: type of setting (urban, semi-urban, and rural); type of facility (primary, secondary, and tertiary); year the HIV care and treatment clinic was established; availability of adolescent-targeted services, including dedicated clinic hours for adolescents, adolescent peer educators, and adolescent support groups; and availability of services likely to be utilized by youth, including screening for sexually transmitted infections, condoms and hormonal contraceptives, and education on high-risk substance abuse behavior.

\section{Outcome definitions}

Pre-antiretroviral therapy outcomes-Cumulative incidence through 1 year after enrollment into HIV care of transferring out to another facility before initiating ART, recorded death before ART initiation, loss to follow-up before ART initiation, and ART initiation was assessed for all patients enrolling into HIV care between January 2005 and September 2010. In the pre-ART period, patients were considered lost to follow-up (LTF) within 1 year if they were not reported as dead or transferred within 1 year and did not make a clinic visit for 1 year with no subsequent visit.

Outcomes after antiretroviral therapy initiation-The subset of patients who initiated ART between January 2005 and March 2011 were assessed for outcome status through 1 year after ART initiation as follows. Cumulative incidence was calculated as the proportion of patients initiating ART who transferred to another facility, were reported death, and became LTF. ART patients were considered LTF within 1 year if they were not 
reported as dead or transferred within 1 year and either were recorded as stopping ART treatment or did not make a clinic visit for 6 months with no subsequent visit. All patients recorded as stopping ART treatment additionally met the visit date-based definition of LTF.

Attrition-The primary outcome was incidence of attrition through 1 year after enrollment in HIV care or ART initiation, depending on the analysis. In both cases, attrition was defined as a documented death or LTF.

\section{Statistical analysis}

Patients were categorized by age at enrollment into HIV care or ART initiation: 10-14 years (young adolescents), 15-24 years (youth), 25-54 years, and at least 55 years. Pre-ARTand ART patients who were LTF were censored at 90 and 15 days after their last visit, respectively, approximating the midpoint between expected visits.

\section{Age comparison of pre-antiretroviral therapy and post-antiretroviral therapy} attrition-For all patients, sub-distribution hazard ratios (sHR) for rates of attrition in the pre-ART period were compared between age groups treating ART initiation and pre-ART attrition as competing risks following the methods outlined by Fine and Grey [18]. Subdistribution hazard ratios present the relative hazard of pre-ART attrition through 1 year after enrollment into care. Among the subset of patients who initiated ART, hazard ratios (HR) for rates of attrition through 1 year after ART initiation were compared between age groups using Cox proportional hazards models. Standard errors were calculated using robust sandwich estimates for variance to account for within-clinic clustering of patients. Both crude and adjusted models were estimated, with adjusted models controlling for sex, pregnancy status, year of enrollment/ ART initiation, point of entry into HIV care (e.g., PMTCT, VCT, PITC, TB/HIV), CD4+ count at enrollment/ART initiation, TB treatment status at enrollment/ART initiation, country, facility type (primary, secondary, tertiary), and clinic location (urban, semi-urban, and rural).

Factors associated with attrition among youth-Among youth, we also identified patient and clinic-level characteristics associated with pre-ART attrition using the methods described above. In addition to examining the association between the factors listed above, we assessed the association between the availability of adolescent-targeted services and availability of services likely to be utilized by youth described earlier. Not all variables measuring availability of adolescent-targeted services had sufficient heterogeneity within country, reducing our ability to jointly adjust for country-level effects and availability of these services. Consequently, multicountry adjusted models included only those services with at least four clinics in each country offering a given service, and at least four clinics in each country not offering a given service. Additional analyses investigated the association between the remaining adolescent-targeted services and attrition in those countries with sufficient heterogeneity.

\section{Results}

A total of 312335 patients at least 10 years of age enrolled into HIV care at the 160 clinics during the observation period, including 147936 who initiated ART. Seventeen percent (53 
244) of the patients enrolling into care, and 10\% (14 844) of the patients initiating ART were youth (Table 1). The proportion of all patients at least 10 years of age who were youth increased over time from $12 \%$ in 2005 to $18 \%$ in 2010, and the proportion of patients initiating ART who were youth increased from $6 \%$ in 2005 to $10 \%$ in 2010. Compared with the overall population, 10 years and older, youth were more likely to be female ( 85 vs. $67 \%$ ), be referred to care from a PMTCT program (20 vs. 9\%), women were more likely to be pregnant at enrollment into care (19 vs. $9 \%$ ) and ART initiation (11 vs. 5\%), and disproportionately more youth were enrolled in Mozambique (70 vs. 56\%). Among the 162 $578(52 \%)$ patients with $\mathrm{CD}^{+}$cell count data available at enrollment into HIV care, youth had higher median $\mathrm{CD}^{+}{ }^{+}$cell counts [383 cells/ $\mu$; interquartile range (IQR): 202-598] comparedwith other age groups [10-14 years: median (IQR) CD4 ${ }^{+}=348(137-640)$ cells $/ \mu \mathrm{l}$; 25-54 years: median $(\mathrm{IQR}) \mathrm{CD}^{+}=251(112-446)$ cells/ $\mu 1$; 255 years: median (IQR) CD4 ${ }^{+}$ $=232(113-414)$ cells $/ \mu 1]$. Among the $100738(68 \%)$ AR patients who had CD4 ${ }^{+}$cell count data available at ART initiation, youth had lower median $\mathrm{CD}^{+}$cell counts $(184 \mathrm{cells} / \mu \mathrm{l}$, IQR: 92-276) comparedwith younger adolescents median (IQR) CD4 ${ }^{+}=204$ (82-338) cells/ $\mu \mathrm{l}]$, and higher $\mathrm{CD} 4^{+}$cell counts compared with other adult age groups [25-54 years: median $(\mathrm{IQR}) \mathrm{CD}^{+}=161(78-244)$ cells/ $\mu \mathrm{l}$; 255 years: median $(\mathrm{IQR}) \mathrm{CD}^{+}=169(93-$ 250) cells/ $\mu \mathrm{l}]$.

One year after enrollment into HIV care, $22 \%$ of all patients were retained in pre-ART care, $42 \%$ had initiated ART, 3\% had transferred to another facility, $2 \%$ were known to have died, and $32 \%$ were LTF. Compared with the overall population, a substantially higher proportion (48\%) of youth were LTF, and a smaller proportion of youth initiated ART (25\%) (Fig. 1a). Among the 140798 patients initiating ART with at least 1 year of potential follow-up (those initiating between January 2005 and September 2010), 70\% of all patients were retained on ART, 7\% transferred to another facility, 5\% were known to have died, and 17\% were LTF 1 year after ART initiation. Compared with the overall population, a substantially higher proportion of youth were LTF (27\%) (Fig. 1b). Among youth, nearly all attrition prior to ART initiation (98\%) and the vast majority after ART initiation (87\%) resulted from LTF.

\section{Attrition before and after antiretroviral therapy initiation by age group}

Table 2 presents crude and adjusted estimates of hazard ratios of attrition before and after ART initiation between youth and other age groups 1 year after enrollment and ART initiation. In adjusted analyses, pre-ART attrition rates 1 year after enrollment into care were higher among youth compared with all other age groups: $\mathrm{sHR}=2.11$ [95\% confidence interval (CI): 1.88-2.37] for youth vs. young adolescents 10-14 years; sHR $=1.50$ (95\% CI: $1.45-1.54$ ) for youth vs. adults aged $25-54$; and sHR $=1.64$ (95\% CI: $1.53-1.75$ ) for youth vs. adults aged at least 55 years. One year after ART initiation, youth also experienced substantially higher attrition compared with other age groups: HR $=2.45$ (95\% CI: $2.15-$ 2.80 ) for youth vs. younger adolescents $10-14$ years; $\mathrm{HR}=1.59$ (95\% CI: 1.52-1.67) for youth vs. adults aged $25-54$, and $\mathrm{HR}=1.62$ (95\% CI: 1.51-1.74) for youth vs. adults aged at least 55 years. Pre-ART and Post-ART LTF was highest among youth compared with other age groups in each of the four countries (data not shown). 


\section{Factors associated with attrition among youth}

Table 3 presents results of multivariate models investigating factors associated with attrition among youth 1 year after enrollment into care and ART initiation. In adjusted pre-ART analyses, nonpregnant female youth had slightly lower attrition compared with male youth ( $\mathrm{sHR}=0.90,95 \%$ CI: 0.86-0.94). Compared with youth entering HIV care after testing HIV positive through voluntary counseling and testing (VCT), youth enrolling in HIV care testing HIV-positive through provider-initiated counseling and testing (PICT) ( $\mathrm{sHR}=1.3$, 95\% CI: 1.2-1.4), TB/HIV clinics (sHR $=1.2,95 \%$ CI: $1.0-1.4$ ), and PMTCT (sHR $=1.3$, 95\% IC: $1.2-1.4$ ) had moderately higher attrition in the pre-ART phase. $\mathrm{CD}^{+}$cell count at enrollment into HIV care was a strong predictor of pre-ART attrition. Youth missing CD4 ${ }^{+}$ cell counts at enrollment [relative risk $(\mathrm{RR})=4.0,95 \% \mathrm{CI}$ : 3.4-4.8], those with $\mathrm{CD}^{+}$cell counts more than 350 cells $/ \mu \mathrm{l}(\mathrm{RR}=2.4,95 \% \mathrm{CI}: 2.1-2.7)$, those with $\mathrm{CD} 4^{+}$cell counts between 200 and 350 cells/ $\mu \mathrm{l}(\mathrm{RR}=1.5,95 \% \mathrm{CI}$ : $1.4-1.7)$, and those with $\mathrm{CD} 4^{+}$cell counts less than 100 cells $/ \mu \mathrm{l}$ at enrollment into HIV care $(\mathrm{RR}=1.2,95 \% \mathrm{CI}: 1.1-1.4)$ experienced higher attrition compared with those enrolling with $\mathrm{CD} 4^{+}$cell counts between 100 and 200 cells $/ \mu$ l. Youth enrolling into HIV care while on treatment for tuberculosis experienced substantially lower attrition in the pre-ART phase compared with youth enrolling not on tuberculosis treatment $(\mathrm{RR}=0.60,95 \% \mathrm{CI}$ : $0.48-0.73$ ). Country was also strongly associated with pre-ART attrition: Youth attending clinics in Kenya $(\mathrm{RR}=2.7,95 \% \mathrm{CI}$ : 1.9-3.9), Mozambique ( $\mathrm{RR}=4.3,95 \% \mathrm{CI}: 3.1-5.9)$, and Tanzania ( $\mathrm{RR}=2.2,1.6-3.3)$ had higher pre-ARTattrition compared with youth attending clinics in Rwanda.

Among clinic-level characteristics assessed, youth attending clinics in semi-urban locations experienced moderately higher attrition $(\mathrm{RR}=1.24,95 \% \mathrm{CI}$ : $1.00-1.53) 1$ year after enrollment in HIV care compared with youth attending clinics in urban settings. Pre-ART attrition was not associated with any of the four adolescent-friendly services assessed with sufficient within-country heterogeneity (adolescent-specific clinic hours, screening for sexually transmitted infections (STIs), provision of condoms, and provision of hormonal contraceptives). In sensitivity analyses restricted to the countries with sufficient heterogeneity to examine whether availability of adolescent support groups, availability of adolescent peer educators, or education on high-risk substance abusing behavior was associated with pre-ART attrition, no significant association was observed (data not shown).

In analyses focusing on attrition among youth 1 year after ART initiation (Table 3), both pregnant $(\mathrm{RR}=0.85,95 \% \mathrm{CI}: 0.74-0.97]$ and nonpregnant $(\mathrm{RR}=0.79,95 \% \mathrm{CI}: 0.73-0.86)$ female youth had lower attrition after ART initiation compared with male youth. Compared with those entering HIV care through VCT, youth initiating ART after identification through PICT ( $R R=1.5,95 \%$ CI: $1.3-1.7$ ) or unknown points of entry ( $R R=1.3,95 \%$ CI: $1.0-1.6)$ had higher attrition, while other points of entry (mostly at clinics other than the enrollment clinic) were not significantly associated with attrition. One year after ART initiation, youth missing $\mathrm{CD}^{+}$cell counts at ART initiation ( $\mathrm{RR}=1.4,95 \% \mathrm{CI}$ : $\left.1.2-1.6\right)$, and those initiating ART with $\mathrm{CD}^{+}{ }^{+}$cell counts more than 350 cells $/ \mu \mathrm{l}(\mathrm{RR}=1.2,95 \% \mathrm{CI}: 0.99-1.5)$, and $\mathrm{CD} 4^{+}$ cell counts less than 100 cells $/ \mu \mathrm{l}(\mathrm{RR}=1.3,95 \% \mathrm{CI}: 1.2-1.5)$ had higher attrition than patients initiating ART with $\mathrm{CD}^{+}$cell counts between 100 and 200 cells $/ \mu$. In contrast to the pre-ART findings, no difference in attrition after ART initiation was observed depending 
on tuberculosis treatment status ( $\mathrm{RR}=1.1,95 \% \mathrm{CI}: 0.81-1.4)$. Similar to the results in the pre-ART analysis, youth attending clinics in Kenya $(\mathrm{RR}=2.5,95 \% \mathrm{CI}: 1.6-3.8)$, Mozambique ( $\mathrm{RR}=4.3$, 95\% CI: 3.3-5.7), and Tanzania $(\mathrm{RR}=2.3,1.5-3.5)$ had higher attrition after ART initiation compared with youth initiating ART in Rwanda.

Among clinic-level factors assessed, there was no significant difference in attrition among youth by facility location or type. Among adolescent-friendly services investigated, clinics providing condoms had substantially lower attrition after ART initiation $(\mathrm{RR}=0.47,95 \%$ CI: 0.32-0.70), while those offering adolescent-specific clinic hours experienced moderately higher attrition $(\mathrm{RR}=1.5,95 \% \mathrm{CI}$ : 1.1-2.1). Screening for STIs and provision of hormonal contraceptives was not associated with attrition after ART initiation in this analysis. In analyses restricted to countries with sufficient variability in availability of adolescent support groups, education on high-risk substance abusing behavior (Kenya, Mozambique, Rwanda), youth attending clinics offering adolescent support groups had lower attrition (RR $=0.73,95 \% \mathrm{CI}: 0.52-1.0$ ), while those attending clinics offering education on high risk substance abusing behavior had higher attrition $(\mathrm{RR}=1.3,95 \% \mathrm{CI}: 1.1-1.5)$. In Rwanda, the sole country with sufficient heterogeneity in the availability of adolescent peer educators, attrition was substantially lower at clinics with this service $(\mathrm{RR}=0.30,95 \% \mathrm{CI}: 0.07-1.3)$, although the confidence interval was wide due to the small clinic sample size.

\section{Discussion}

As the first multicountry and largest study to date on attrition among HIV-infected youth enrolled in HIV care and on ART in sub-Saharan Africa, this study provides important insights on program outcomes previously not sufficiently described. Specifically, in addition to confirming attrition 1 year after ART initiation is highest among youth compared with all other age groups, as shown in other studies [11-17], we found that youth were substantially more likely than young adolescents and older adults to die or be lost to follow-up before initiating ART. These findings, across a large and diverse study population covering 160 clinics and four countries in sub-Saharan Africa, held after adjustment for important patientlevel and clinic-level factors.

Eighty-five percent of youth in our study population were female, on par with the sex distribution observed among adolescents and young adults in other studies in Africa $[11,13,19,20]$. In our study, $19 \%$ of female youth were pregnant at enrollment into HIV care, and $20 \%$ enrolled through PMTCT, highlighting an important point of entry into clinical care for this population. Youth also enrolled into HIV care on average healthier than the younger and older adult patient population. Our observation of high attrition among youth compared with other age groups after adjustment for baseline markers of health $\left(\mathrm{CD} 4^{+}\right.$cell count and tuberculosis treatment status) suggests that youth may be becoming LTF for reasons other than illness. High levels of migration for work [21], and lack of belief in the benefits of attending clinic and initiating ART among youth [22], particularly if healthy and not yet eligible to initiate ART, are two potential explanations for these findings.

Our findings also indicate a potential benefit of certain services targeting young people on attrition, particularly after ART initiation. One hypothesis why such services were not 
associated with improved pre-ART retention may be that youth lost to follow-up in the preART phase tend to be lost very soon after enrollment, and may not have had the opportunity to engage in the adolescent-friendly services offered at the clinic. After ART initiation, youth attending clinics that offered condoms and adolescent support groups experienced substantially lower attrition after ART initiation than youth attending clinics not offering these services. Further, among youth in Rwanda, those attending clinics with adolescent peer educators experienced lower attrition than those attending clinics not offering this service. Further analyses, including qualitative analyses, are needed to explore why certain services were associated with improved attrition, and certain services were not.

Our study has several important strengths. To our knowledge, it is the largest and sole multicountry study investigating attrition among youth enrolled in HIV care and treatment in sub-Saharan Africa. Additionally, it is the first to examine outcomes both before and after ART initiation, as well as the first to assess whether availability of services targeting young people is associated with a decreased risk of attrition among youth. Use of data collected as part of routine care, rather than in a research setting, also strengthens our study to investigate current conditions at facilities typical of those wherein a majority of individuals seek HIV care.

A few limitations must also be noted. A large proportion of patients were missing data on $\mathrm{CD}^{+}$cell count, an important risk factor for attrition. Given the likelihood that patients missing $\mathrm{CD}^{+}$cell counts differ systematically from those with complete data, we included a 'missing' $\mathrm{CD}^{+}$cell count category in all analyses, rather than imputing these data. Second, when investigating the association between services targeting adolescents and attrition, we were unable to completely separate out the effect of these services from more general between-country differences in attrition because not all services had sufficient withincountry heterogeneity. Analyses described in this article remove countries with insufficient heterogeneity from analyses, but caution is warranted in generalizing these results, as we cannot rule out that some of the associations between adolescent-targeted services and attrition are due to confounding by country effects. Additionally, information on the availability of adolescent-friendly services was obtained in 2011, after follow-up of the patients in the study. We expect that clinics initiated rather than terminated adolescentfriendly services during the study period, leading to potential over-estimation of the proportion of patients enrolled in HIV care at clinics with such services. This likely biases our measures of the association between availability of adolescent-friendly services and attrition toward the null, and thus our results should be considered lower bound estimates. Further, as data were available on services targeting adolescents rather than youth, we assumed that adolescent-friendly services were also used by youth. Finally, as we had no information on means of HIV acquisition, we were unable to assess whether risk factors for attrition vary between perinatally infected youth and those who acquired HIV behaviorally.

In conclusion, we show that youth accessing HIV care services in 160 clinics across four sub-Saharan African countries severely affected by the HIV epidemic are at substantially higher risk of attrition than younger adolescents and older adults both before and after ART initiation, and that adolescent-friendly services may beneficially impact retention among youth after ART initiation. To enhance retention in this important and growing population, 
we suggest that youth-friendly interventions be implemented and evaluated for their effectiveness in improving retention.

\section{Acknowledgments}

This work was supported by the Identifying Optimal Models of HIV Care and Treatment Study (Centers for Disease Control and Prevention); Grant Number 5U2GPS001537-03.

\section{References}

1. UNICEF. Opportunity in crisis: preventing HIV from early adolescence to young adulthood. New York: United Nations; 2011.

2. United Nations. World Population Prospects: the 2008 Revision, Highlights. New York: United Nations; 2009.

3. UNAIDS. , editor. UNAIDS. World AIDS day report 2012. Geneva: UNAIDS; 2012.

4. Ferrand RA, Corbett EL, Wood R, Hargrove J, Ndhlovu CE, Cowan FM, et al. AIDS among older children and adolescents in Southern Africa: projecting the time course and magnitude of the epidemic. AIDS. 2009; 23:2039-2046. [PubMed: 19684508]

5. McNairy ML, El-Sadr WM. The HIV care continuum: no partial credit given. AIDS. 2012; 26:1735-1738. [PubMed: 22614888]

6. Cherutich P, Kaiser R, Galbraith J, Williamson J, Shiraishi RW, Ngare C, et al. Lack of knowledge of HIV status a major barrier to HIV prevention, care and treatment efforts in Kenya: results from a nationally representative study. PLoS One. 2012; 7:e36797. [PubMed: 22574226]

7. Conserve D, Sevilla L, Mbwambo J, King G. Determinants of previous HIV testing and knowledge of partner's HIV status among men attending a voluntary counseling and testing clinic in Dar es Salaam, Tanzania. Am J Men Health. 2012 Epub ahead of print. 10.1177/1557988312468146

8. Hutchinson PL, Mahlalela X. Utilization of voluntary counseling and testing services in the Eastern Cape, South Africa. AIDS care. 2006; 18:446-455. [PubMed: 16777636]

9. Tabana H, Doherty T, Swanevelder S, Lombard C, Jackson D, Zembe W, et al. Knowledge of HIV status prior to a community HIV counseling and testing intervention in a rural district of south Africa: results of a community based survey. BMC Infect Dis. 2012; 12:73. [PubMed: 22458410]

10. Ferguson J, Dick B, Ross DA. Preventing HIV/AIDS in young people a systematic review of the evidence from developing countries conclusions and recommendations. World Health Organ Tech Rep Ser. 2006; 938:1-13. discussion 317-341. [PubMed: 16921915]

11. Nachega JB, Hislop M, Nguyen H, Dowdy DW, Chaisson RE, Regensberg L, et al. Antiretroviral therapy adherence, virologic and immunologic outcomes in adolescents compared with adults in southern Africa. J Acquir Immune Defic Syndr. 2009; 51:65-71. [PubMed: 19282780]

12. Bakanda C, Birungi J, Mwesigwa R, Nachega JB, Chan K, Palmer A, et al. Survival of HIVinfected adolescents on antiretroviral therapy in Uganda: findings from a Nationally Representative Cohort in Uganda. PLoS One. 2011; 6:e19261. [PubMed: 21559441]

13. Weigel R, Estill J, Egger M, Harries AD, Makombe S, Tweya H, et al. Mortality and loss to follow-up in the first year of ART: Malawi national ART programme. AIDS. 2012; 26:365-373. [PubMed: 22095194]

14. Wandeler G, Keiser O, Pfeiffer K, Pestilli S, Fritz C, Labhardt ND, et al. Outcomes of antiretroviral treatment programs in rural Southern Africa. J Acquir Immune Defic Syndr. 2012; 59:e9-16. [PubMed: 22067665]

15. Jani IV, Sitoe NE, Alfai ER, Chongo PL, Quevedo JI, Rocha BM, et al. Effect of point-of-care CD4 cell count tests on retention of patients and rates of antiretroviral therapy initiation in primary health clinics: an observational cohort study. Lancet. 2011; 378:1572-1579. [PubMed: 21951656]

16. Ekouevi DK, Balestre E, Ba-Gomis FO, Eholie SP, Maiga M, Amani-Bosse C, et al. Low retention of HIV-infected patients on antiretroviral therapy in 11 clinical centres in West Africa. Trop Med Int Health. 2010; 15(Suppl 1):34-42. [PubMed: 20586958] 
17. Boyles TH, Wilkinson LS, Leisegang R, Maartens G. Factors influencing retention in care after starting antiretroviral therapy in a rural South African programme. PLoS One. 2011; 6:e19201. [PubMed: 21559280]

18. Fine JP, Gray RJ. A proportional hazards model for the subdistribution of a competing risk. J Am Stat Assoc. 1999; 94:496-509.

19. Hawkins C, Chalamilla G, Okuma J, Spiegelman D, Hertzmark E, Aris E, et al. Sex differences in antiretroviral treatment outcomes among HIV-infected adults in an urban Tanzanian setting. AIDS. 2011; 25:1189-1197. [PubMed: 21505309]

20. Mills EJ, Bakanda C, Birungi J, Chan K, Hogg RS, Ford N, et al. Male gender predicts mortality in a large cohort of patients receiving antiretroviral therapy in Uganda. J Int AIDS Soc. 2011; 14:52. [PubMed: 22050673]

21. United Nations Department of Economic and Social Affairs. International migration in a globalizing world: the role of youth. New York: United Nations; 2011.

22. World Health Organization. Retention in HIV programmes: Defining the challenges and identifying solutions. Geneva: World Health Organization; 2012. 
(a)

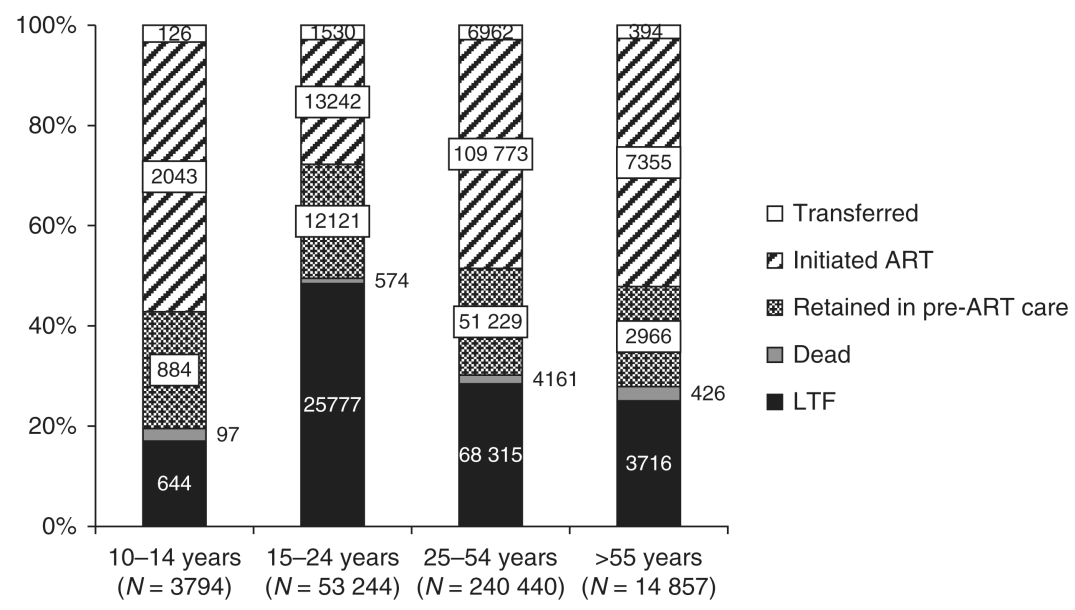

(b)

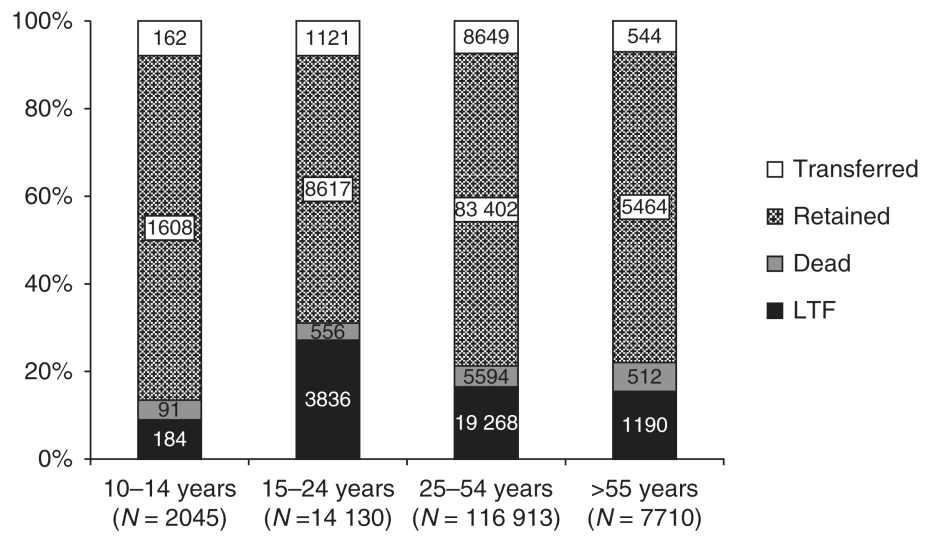

Fig. 1. (a) Patient status 1 year after enrollment into HIV care, by age group. (b) Patient status 1 year after antiretroviral therapy (ART) initiation, by age group 


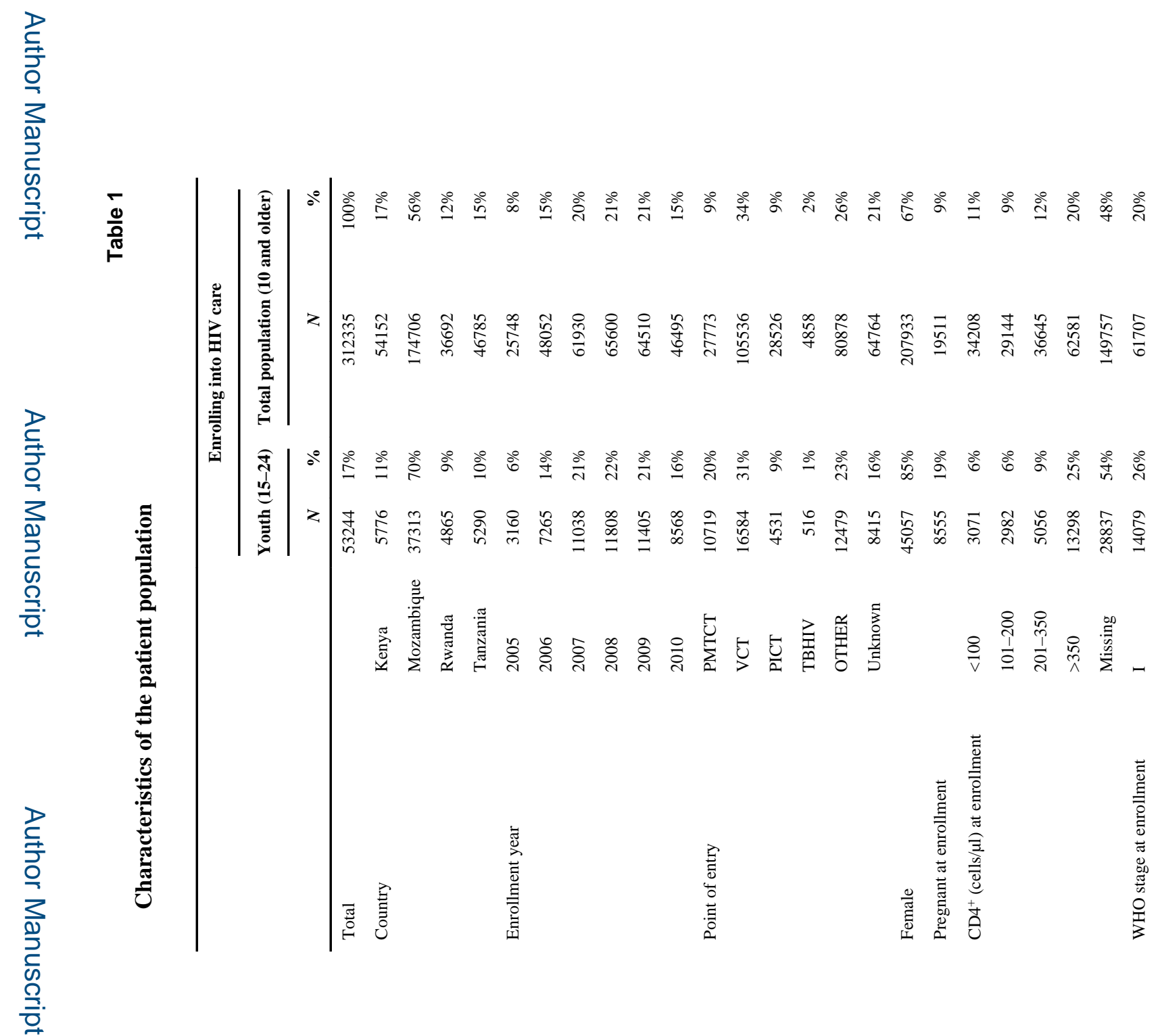
AIDS. Author manuscript; available in PMC 2015 July 28. 


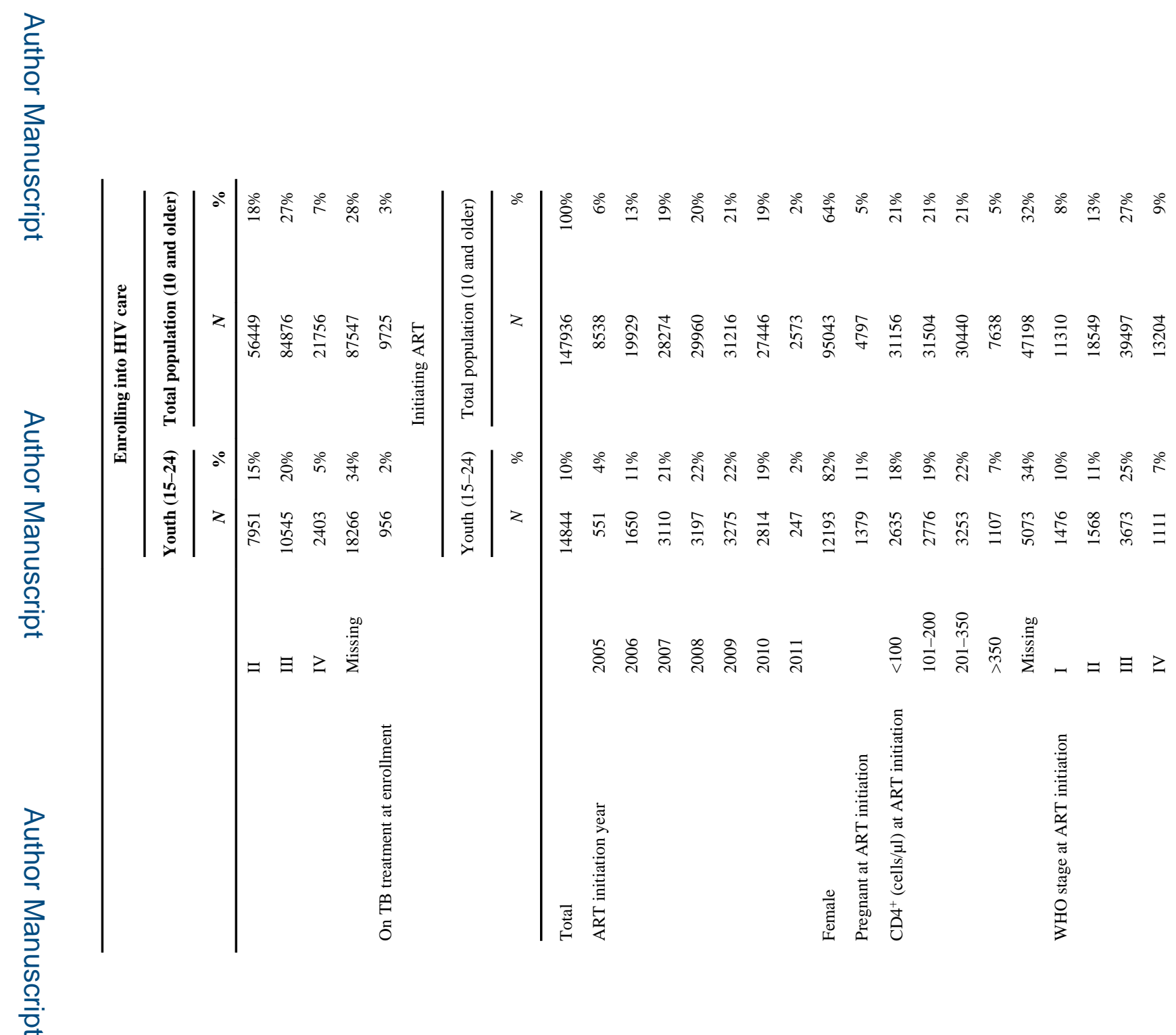



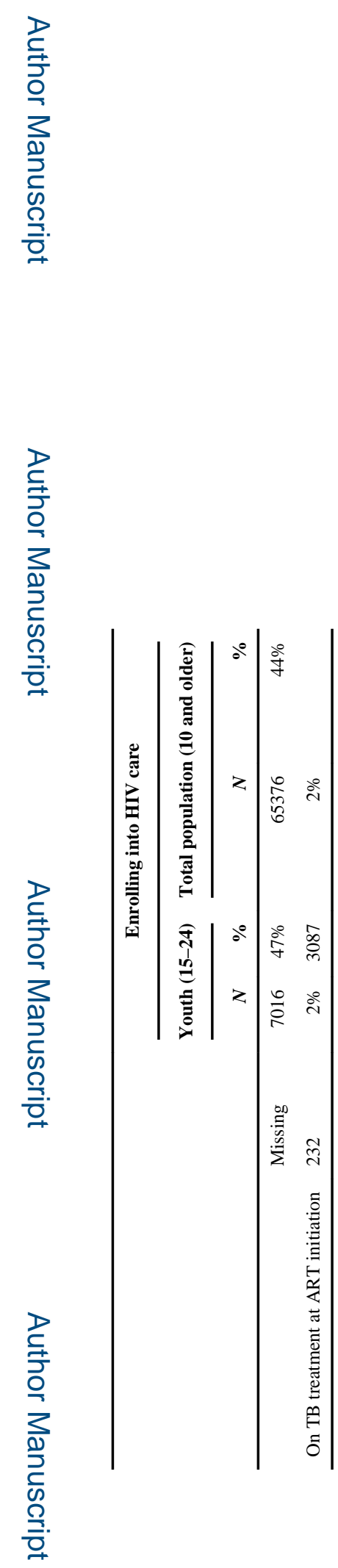

AIDS. Author manuscript; available in PMC 2015 July 28. 


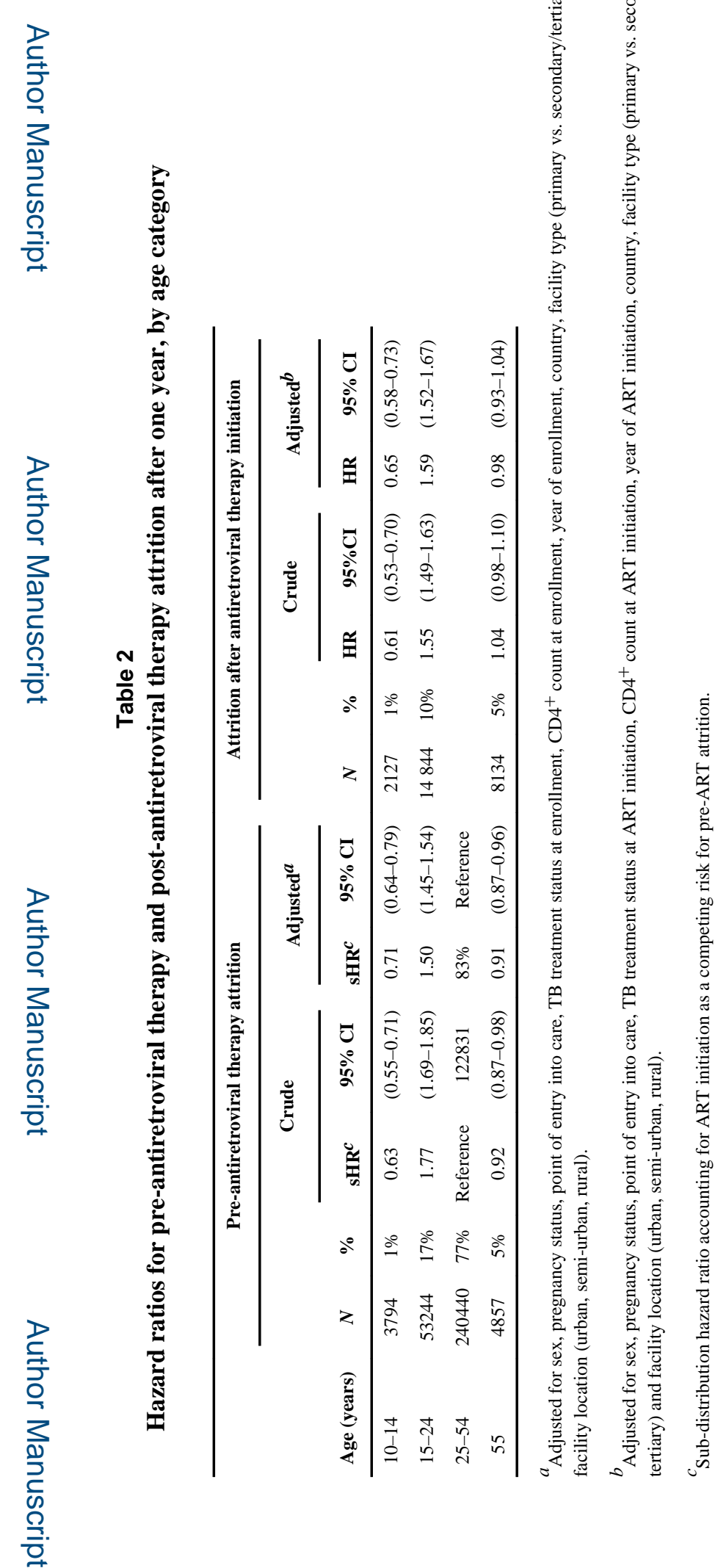

AIDS. Author manuscript; available in PMC 2015 July 28. 


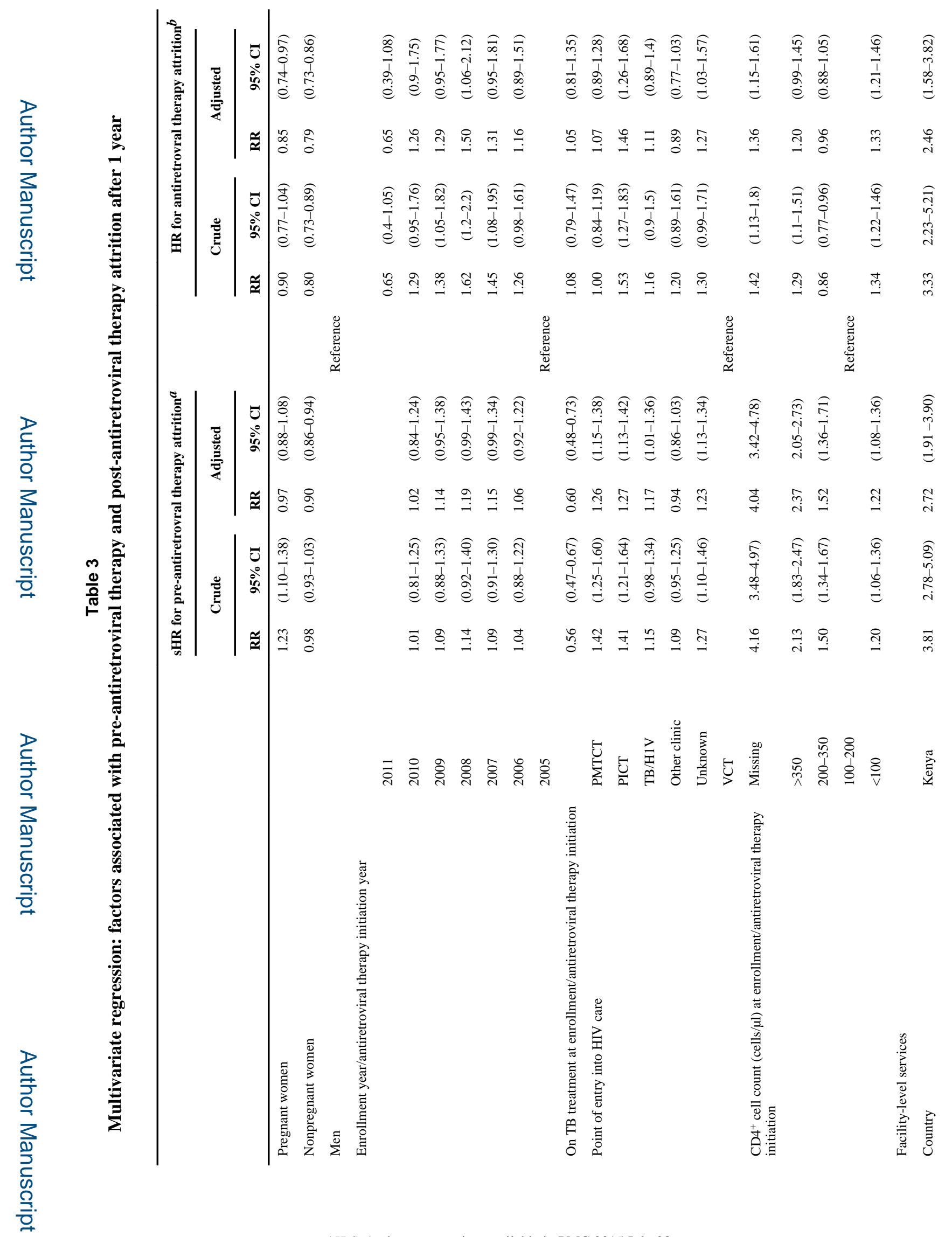

AIDS. Author manuscript; available in PMC 2015 July 28. 
Lamb et al.

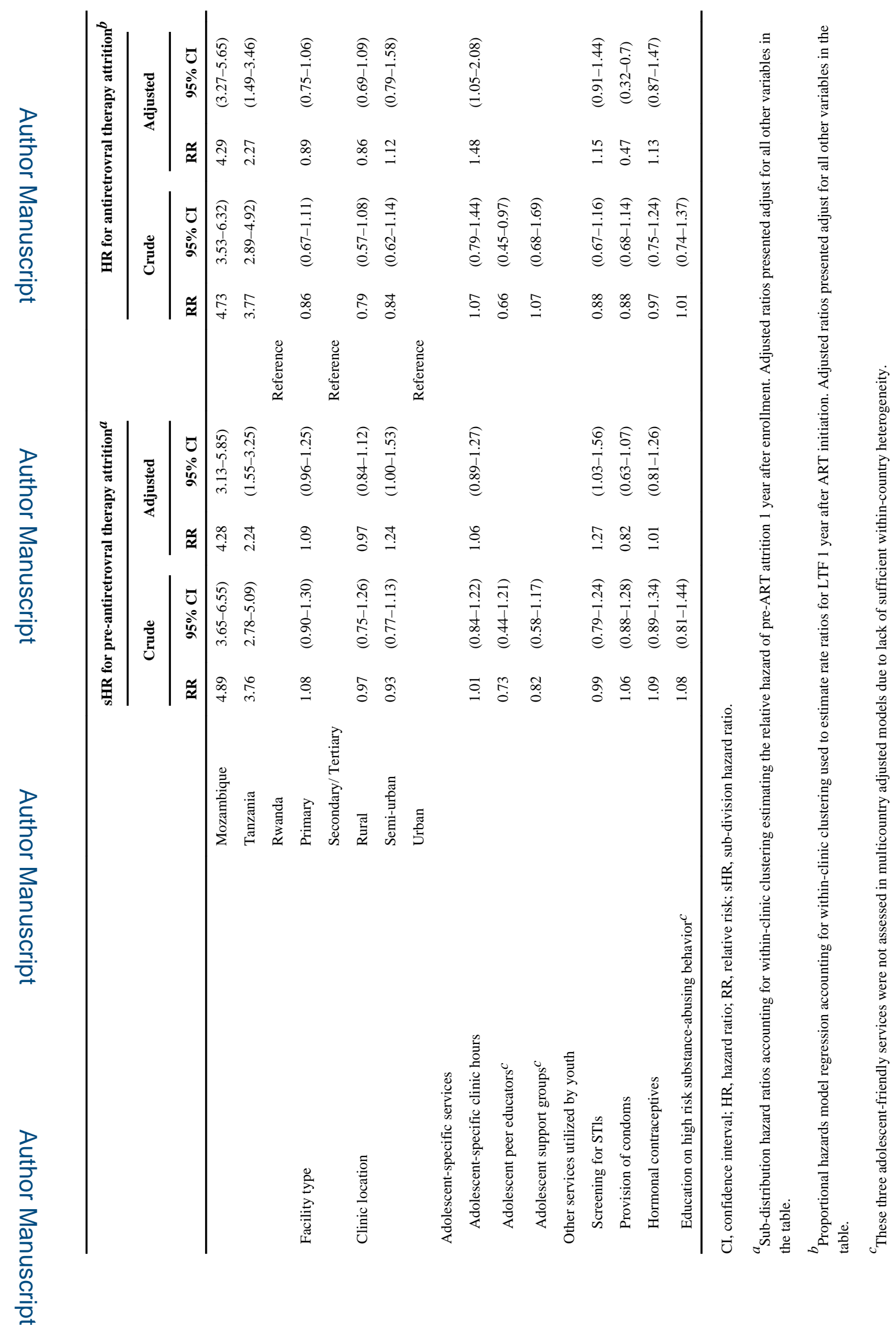

AIDS. Author manuscript; available in PMC 2015 July 28. 